\title{
Vehicles instability criteria for flood risk assessment of a street network
}

\author{
Chiara Arrighi ${ }^{1}$, Nicolas Huybrechts ${ }^{2,3}$, Abdellatif Ouahsine ${ }^{2}$, Patrick Chassé ${ }^{3}$, Hocine Oumeraci ${ }^{4}$, and \\ Fabio Castelli ${ }^{1}$ \\ ${ }^{1}$ Department of Civil and Environmental Engineering, University of Florence, Florence, Italy \\ ${ }^{2}$ Laboratoire Roberval, Sorbonne Universités, Université de Technologie de Compiègne, CNRS, Centre de \\ Recherches de Royallieu, Compiègne Cedex, France \\ ${ }^{3}$ CEREMA-DTecEMF, Margny Lès Compiègne, France \\ ${ }^{4} \mathrm{TU}$ Brauschweig, Leicthweiss Institute for Hydraulic Engineering and Water Resources, Braunschweig, \\ Germany
}

Correspondence to: Chiara Arrighi (chiara.arrighi@dicea.unifi.it)

Published: 12 May 2016

\begin{abstract}
The mutual interaction between floods and human activity is a process, which has been evolving over history and has shaped flood risk pathways. In developed countries, many events have illustrated that the majority of the fatalities during a flood occurs in a vehicle, which is considered as a safe shelter but it may turn into a trap for several combinations of water depth and velocity. Thus, driving a car in floodwaters is recognized as the most crucial aggravating factor for people safety. On the other hand, the entrainment of vehicles may locally cause obstructions to the flow and induce the collapse of infrastructures. Flood risk to vehicles can be defined as the combination of the probability of a vehicle of being swept away (i.e. the hazard) and the actual traffic/parking density, i.e. the vulnerability. Hazard for vehicles can be assessed through the spatial identification and mapping of the critical conditions for vehicles incipient motion. This analysis requires a flood map with information on water depth and velocity and consistent instability criteria accounting for flood and vehicles characteristics. Vulnerability is evaluated thanks to the road network and traffic data. Therefore, vehicles flood risk mapping can support people's education and management practices in order to reduce the casualties. In this work, a flood hazard classification for vehicles is introduced and an application to a real case study is presented and discussed.
\end{abstract}

\section{Introduction}

According to global statistics, in the last decade floods affected the largest number of people with respect to other natural hazards (EM-DAT, 2012). In developed countries the majority of fatalities occurs in vehicles (Jonkman and Kelman, 2005; Maples and Tiefenbacher, 2009; Fitzgerald et al., 2010; Kellar and Schmidlin, 2012). On one hand, vehicles can become unstable by losing traction even for very low water depths, or they become buoyant, so that they are swept away by floodwaters and possibly produce a debris. On the other hand, several fatalities occur for inappropriate high-risk behaviours, like driving in flooded streets. These are the reasons why vehicles are usually recognized as one of the most aggravating factors in urban floodings (Rodriguez et al., 2006; Franklin et al., 2014). Although the problem of vehicle mobilization is so crucial, only a few experimental investigation on the incipient motion conditions of small-scale vehicle models have been carried out so far (Shu et al., 2011, Xia et al., 2011, 2014). The experimental data on vehicles instability, expressed as pairs of water depth and velocity, are affected by a large scatter, which depends on the different characteristics (i.e. weight, shape, height of the planform) of the considered vehicle model.

Moreover, the different contribution of the hydrodynamic forces and the influence of flow regime on the mechanism of the onset of motion has been numerically studied by Arrighi et al. (2015). 
The aim of the work is to introduce hazard criteria for vehicles at rest in flooded streets easily applicable to inundation maps, which account for both flood and vehicles characteristics, in order to overcome the scatter of existing experimental data.

\section{Dimensionless instability diagram for flooded vehicles}

Incipient motion for sliding occurs when the drag force exerted by the water just exceeds the friction force, which is the product of the friction coefficient $\mu$ and immersed weight of the car (Eq. 1). The onset of motion by sliding of a vehicle is modeled using the geometric scheme shown in Fig. 1a. The flow direction has an orientation $\beta$ with respect to the longitudinal axis of the car and the bed slope is assumed null, the wheels are considered as locked.

$$
\begin{aligned}
& \frac{1}{2} \rho C_{\mathrm{D}} U^{2} A_{\mathrm{D}}>\mu\left[\rho_{\mathrm{c}} g\left(H_{\mathrm{V}}-h_{\mathrm{c}}\right) l L-\rho g\left(H-h_{\mathrm{c}}\right) l L\right. \\
& \left.\quad-\frac{1}{2} \rho C_{\mathrm{L}} U^{2} A_{\mathrm{L}}\right]
\end{aligned}
$$

where $\rho$ is the water density, $\rho_{\mathrm{c}}$ is the car density, $U$ is the mean flow velocity, $H$ is the water depth, $H_{\mathrm{V}}$ is the height of the vehicle, $h_{\mathrm{c}}$ is the height of the planform, $g$ is the acceleration of gravity, $l$ and $L$ are the car width and length and $C_{\mathrm{D}}$ and $C_{\mathrm{L}}$ are the drag and lift coefficients (official IS of units). The reference areas for drag $\left(A_{\mathrm{D}}\right)$ and lift $\left(A_{\mathrm{L}}\right)$ forces are the projection of the full area of the car normal to the flow (Eq. 2) and the planform (Eq. 3) which are respectively

$A_{\mathrm{D}}=(l \cdot \cos \beta+L \cdot \sin \beta) \cdot\left(H_{\mathrm{V}}-h_{\mathrm{c}}\right)$

$A_{\mathrm{L}}=L \cdot l$

The manipulation of Eq. (1) here modified to account for any given flow orientation after Arrighi et al. (2015) yields a relation between the square of Froude number $\frac{U^{2}}{g H}$ and a dimensionless group of variables which is called mobility parameter $\theta_{\mathrm{V}}$

$\theta_{\mathrm{V}}=\frac{2 L}{\left(H_{\mathrm{V}}-h_{\mathrm{c}}\right)} \cdot \alpha \cdot\left(\frac{\rho_{\mathrm{c}} \cdot\left(H_{\mathrm{V}}-h_{\mathrm{c}}\right)}{\rho \cdot\left(H-h_{\mathrm{c}}\right)}-1\right)$

$\alpha=\frac{l}{l \cdot \cos \beta+L \cdot \sin \beta}$

where the first term is a shape factor, $\alpha$ accounts for the angle of flow incidence and the latter accounts for the immersed weight of the car.

The mobility parameter has been calculated for three experimental datasets (Shu et al., 2011, Xia et al., 2011, 2014) including seven different car models and densities and three model scales $(1: 14,1: 18,1: 43)$. The values of the calculated mobility parameter $\theta_{\mathrm{V}}$ plotted against Froude number identify a unique threshold of incipient motion (Fig. 1c),

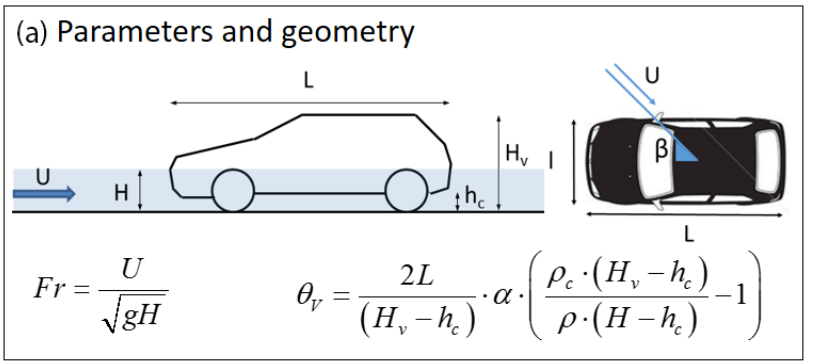

(b) Hazard criterion

$$
\frac{\theta_{V c r}}{\theta_{V}}\left\{\begin{array}{lll}
>1 & \text { Motion } \longrightarrow & \text { danger } \\
=1 & \text { Incipient motion } & \\
<1 & \text { No motion } \longrightarrow & \text { safety }
\end{array}\right.
$$

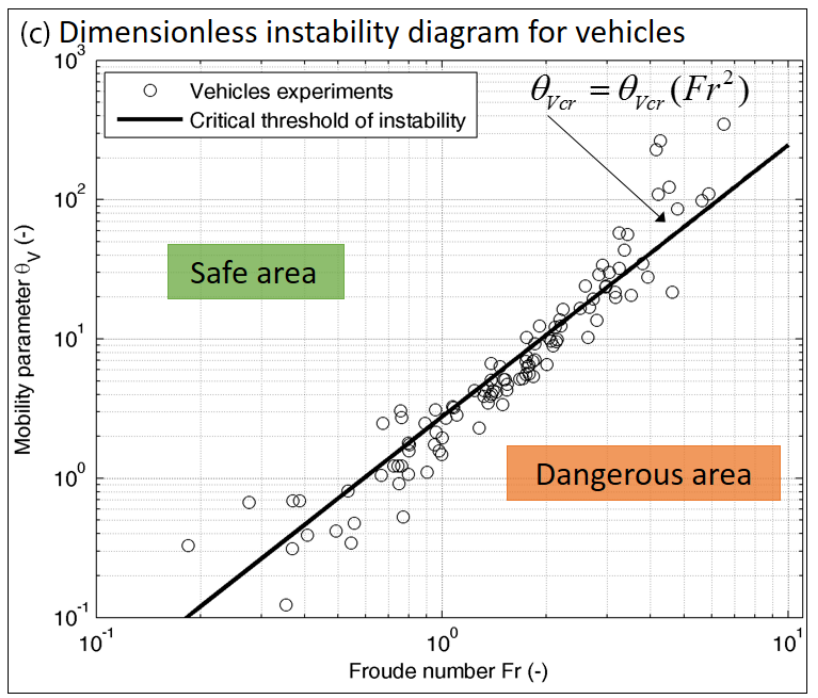

Figure 1. Sketch of the car geometry and definition of the mobility parameter (a), definition of hazard criterion (b) and dimensionless instability diagram for flooded vehicles with identification of the critical threshold (c) (experimental data by Shu et al., 2011; Xia et al., 2011, 2014).

which separates safe conditions (above the curve) from dangerous conditions (below the curve) where the vehicle mobilization is likely to occur. Thus, a hazard criterion can be defined as the ratio between the critical threshold $\theta_{\mathrm{v}_{\mathrm{cr}}}$ and the mobility parameter $\theta_{\mathrm{V}}$ for a predefined reference vehicle and flow regime (i.e. water depth and velocity of an inundation map) as shown in Fig. 1b.

\section{The case study of Ajaccio (France)}

The study area considered to show the application of the vehicles hazard criterion derived from the mobility parameter, is the Les Cannes district in Ajaccio, situated in southwestern Corse (France). The flood simulation has been carried out with TELEMAC-2D (Galland et al., 1991) solving the shallow water equations over an unstructured grid 
of triangular elements. The results have been provided by CEREMA (Centre d'études et d'Expertise sur les Risques, l'Environment, la Mobilité et l'Aménagement) and are described in detail in a project report for the Direction Départementale de l'Equipment Corse du Sud (Sogreah, 2006).

The Cannes district is located downstream of three small catchments (Arbitrone, Moulins Blancs and Arbajola), whose streams are culverted in the urbanized area up to the gulf (Fig. 2, top). The cadastral map 1:5000 has been used to extract the built footprint and a detailed topographic survey has been carried out to measure the terrain elevation ( $\max 30 \mathrm{~m}$ a.s.l.). The mesh is composed of 10511 nodes and 18486 elements, with edge size ranging from 0.5 to $25 \mathrm{~m}$ in the sea.

Although the area is not densely urbanized, it hosts two educational facilities and some commercial activities and it is connected to the port area through an important traffic artery along the coastline, the Cours Jean Nicoli.

Benchmark inundation events date back to 23 January 2003 and 25-26 November 1990 and allowed the validation of the numerical model (Sogreah, 2006).

The maximum water depth reached in the Cannes district for the simulated 100 years scenario is about $2 \mathrm{~m}$ (Fig. 2, top) and the maximum velocity is above $3.0 \mathrm{~m} \mathrm{~s}^{-1}$. The dark blue area at the bottom of the top panel in Fig. 2 represents the sea level used as a southern boundary condition. The hazard level is calculated for a Ford Focus and $\beta=90^{\circ}$ (i.e. a medium size passenger vehicle oriented normally to the flow) combining in each computational cell, for each time step, the variables obtained during the simulation (i.e. water depth and velocity). Figure 2 (bottom) shows the extraction of the maximum value of the vehicle hazard over the simulation time. The colour scale goes from 0.25 (green colour, very low hazard) up to 1.5 (dark violet, very high hazard). Red areas are those likely to be prone to incipient motion of vehicles (high hazard) and violet areas can be considered as extremely dangerous for parked vehicles since the hazard level is higher than one. Moreover, since many open areas are used as car parking for the commercial areas, it is likely that vehicles can be swept away by floodwaters for a similar scenario.

\section{Conclusions}

The mobility parameter allows defining hazard criteria capable of accounting for both flood and vehicles characteristics. The hazard map here presented can be drawn for different probability scenarios and can be used to assess the risk for vehicles if the parking/traffic density is accounted for. From this preliminary test, the main traffic artery shows very high hazard levels for the simulated 100 years flood and can be significantly affected in case of event.

The available visual documentation of the 2003 flood event demonstrate that vehicles mobilization occurred in the area. The presence of the schools is a further traffic-attractor

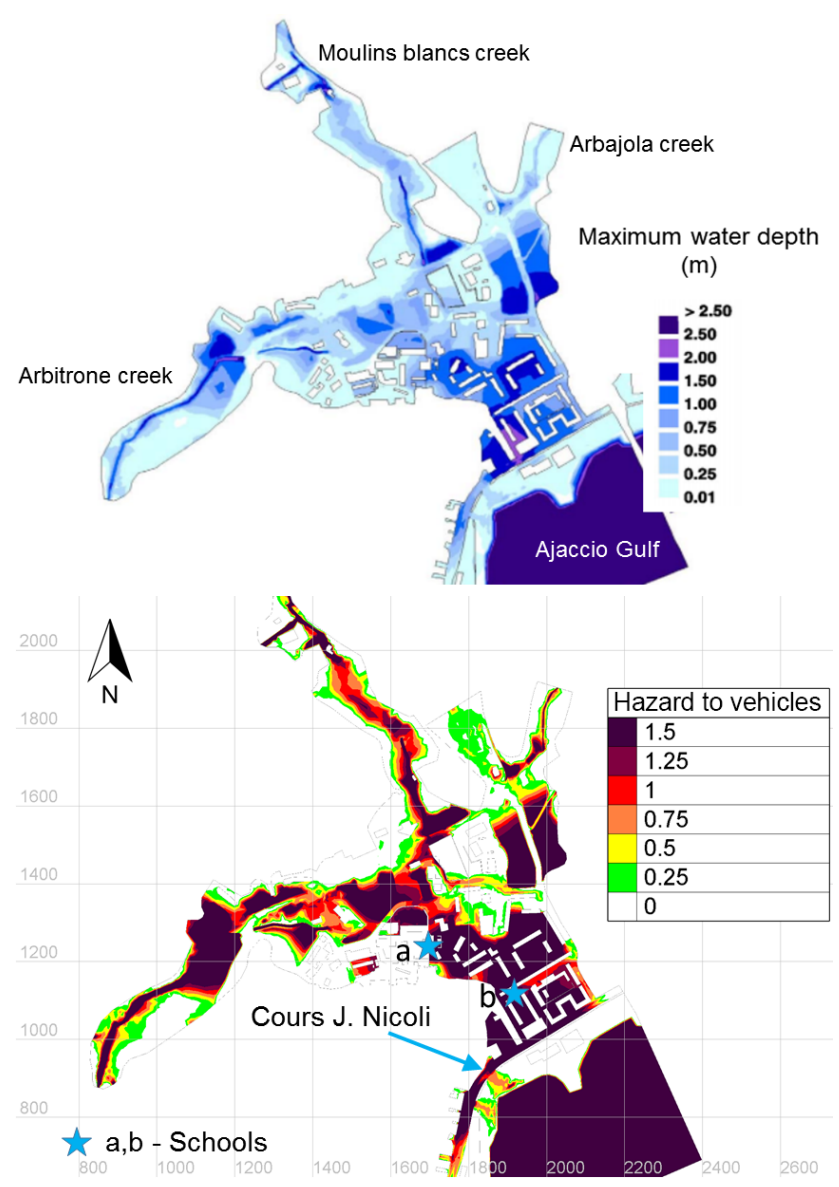

Figure 2. Flood depth map for the simulated 100 years scenario (top) and temporal maximum of the hazard for vehicles with identification of vulnerable buildings and main traffic artery (bottom).

during several time slots and represents a vulnerability factor. In order to prevent people's fatalities, hazard maps for vehicles can be adopted to implement non-structural flood risk mitigation measures (i.e. people education, traffic management) or emergency actions (roads closure).

\section{References}

Arrighi, C., Alcèrreca-Huerta, J. C., Oumeraci, H., and Castelli, F.: Drag and lift contribution to the incipient motion of partly submerged flooded vehicles, J. Fluids Structures, 57, 170-184, 2015.

EM-DAT: Disasters in numbers (October 2000), available at: http: //www.emdat.be/publications, 2012.

Fitzgerald, G., Du, W., Jamal, A., Clark, M., and Hou, X. Y.: Flood fatalities in contemporary Australia (1997-2008), Em. Med. Australasia, 22, 180-186, 2010.

Franklin, R. C. King, J. C., Aitken, P. J., and Leggat, P. A.: "Washed away"-assessing community perceptions of flooding and prevention strategies: A North Queensland example, Nat. Haz., 73, 1977-1998, 2014. 
Galland, J. C., Goutal, N., and Hervouet, J. M.: TELEMAC: A new numerical model for solving shallow water equations, Adv. Water Res., 14, 138-148, 1991.

Jonkman, S. N. and Kelman, I.: An analysis of the causes and circumstances of flood disaster deaths, Disasters, 29, 75-97, 2005.

Kellar, D. M. M. and Schmidlin, T. W.: Vehicle-related flood deaths in the United States, 1995-2005, J. Flood Risk Manage., 5, 153$163,2012$.

Maples, L. and Tiefenbacher, J.: Landscape, development, technology and drivers: The geography of drownings associated with automobiles in Texas floods, 1950-2004, Appl. Geogr., 29, 224234, 2009.

Rodriguez, H., Quarantelli, E. L., and Dynes, R. R.: Handbook of Disaster Research, Springer Science, 2006.
Sogreah: Direction Départementale de l'Equipment Corse du Sud. Evaluation du risqué pluvial sur la commune d'Ajaccio-Etude hydraulique dans les bassins versant d'Arbitronne, San Remedio at la Madonnuccia, No. 274 0190, 2006.

Shu, C., Xia, J., Falconer, R., and Lin, B.: Estimation of incipient velocity for partially submerged vehicles in floodwaters,J. Hydrol. Res., 49, 709-717, 2011.

Xia, J., Teo, F. Y., Lin, B., and Falconer, R.: Formula of incipient velocity for flooded vehicles, Nat. Haz., 58, 1-14, 2011.

Xia, J., Falconer, R. A., Xiao, X., and Wang, Y.: Criterion of vehicle stability in floodwaters based on theoretical and experimental studies, Nat. Haz, 70, 1619-1630, 2014. 